\title{
Time-resolved imaging techniques applied to femtosecond laser material processing
}

\section{Técnicas de imagen con resolución temporal aplicadas al procesado de materiales con pulsos láser ultracortos}

\author{
Mario Garcia-Lechuga ${ }^{1} \mathrm{~S}^{*}$ \\ 1. Departamento de Física Aplicada, Universidad Autónoma de Madrid, Campus de Cantoblanco, 28049 \\ Madrid, Spain \\ (`) E-mail: mario.garcialechuga@uam.es \\ S: miembro de SEDOPTICA / SEDOPTICA member
}

\author{
Received: 20/03/2021 Accepted: 10/05/2021
}

DOI: $10.7149 /$ OPA.54.2.51059

\begin{abstract}
:
This article summarizes the motivation, the experimental procedures and the main results of the thesis awarded with the $7^{\text {th }}$ Justianiano Casas Award. Within the thesis, different strategies based on timeresolved microscopy were developed and applied to the study of changes occurring at the surface of materials upon femtosecond laser excitation. First, this optical pump-and-probe technique is applied to the study of the ablation dynamics of lithium niobate, a crystalline dielectric material with numerous applications in optics and photonics. Second, the transformation dynamics of a phosphate glass was investigated upon irradiation with sub-ablative laser pulse fluences, leading to the formation of a permanent heat affected layer. To this end, a fiber-based optical delay line was developed, enabling to explore delays up to $0.5 \mu \mathrm{s}$. Third and last, an optical method capable of simultaneously fabricating laser-induced periodic structures and resolving spatially and temporally their formation process is reported. The results presented in this thesis demonstrate the huge potential of time-resolved pumpprobe microscopy for revealing the process dynamics of a multitude of material modification processes upon femtosecond laser excitation.
\end{abstract}

Key words: laser material processing, microscopy, femtosecond lasers

\section{RESUMEN:}

En este artículo se resume la motivación, los procedimientos experimentales y los principales resultados de la tesis premiada con el VII Premio Justianiano Casas. En esta tesis se desarrollaron y aplicaron diferentes estrategias basadas en la microscopía resuelta en el tiempo para el estudio de los cambios superficiales inducidos en los materiales tras la excitación con pulsos láser de femtosegundo. En primer lugar, esta técnica de bombeo-muestreo se aplicó al estudio de la dinámica de ablación del niobato de litio, un material dieléctrico cristalino con numerosas aplicaciones en óptica y fotónica. En segundo lugar, se investigó la dinámica de transformación de un vidrio de fosfato al ser irradiado por debajo de su umbral de ablación, condiciones en las que se forma una capa permanente afectada por el calor. Para este estudio se desarrolló una línea de retardo óptica basada en fibra, que permite explorar retardos de hasta 0,5 $\mu$ s. En tercer y último lugar, se muestra un método capaz de fabricar estructuras periódicas y simultáneamente observar su evolución espacial y temporal. Los resultados presentados en esta tesis demuestran el enorme potencial de la microscopía de sonda de bombeo resuelta en el tiempo para revelar la dinámica del proceso de una multitud de procesos de modificación de materiales tras la excitación con láser de femtosegundo.

Palabras clave: procesado de materiales con láser, microscopía, láseres de femtosegundo 


\section{REFERENCES AND LINKS / REFERENCIAS Y ENLACES}

[1] K. Plamann, F. Aptel, C. L. Arnold, A. Courjaud, C. Crotti, F. Deloison, F. Druon, P. Georges, M. Hanna, J.M. Legeais, F. Morin, É. Mottay, V. Nuzzo, D. A. Peyrot, and M. Savoldelli, "Ultrashort pulse laser surgery of the cornea and the sclera," J. Opt. 12, 084002 (2010).

[2] M. Farsari and B. N. Chichkov, "Materials processing: Two-photon fabrication," Nat. Photonics 3, 450452 (2009).

[]ㅡ Z.-Z. Li, L. Wang, H. Fan, Y.-H. Yu, Q.-D. Chen, S. Juodkazis, and H.-B. Sun, "O-FIB: far-field-induced nearfield breakdown for direct nanowriting in an atmospheric environment," Light Sci. Appl. 9, 41 (2020).

[4] P. Balling and J. Schou, "Femtosecond-laser ablation dynamics of dielectrics: basics and applications for thin films.," Rep. Prog. Phys. 76, 036502 (2013).

[5] M. Malinauskas, A. Žukauskas, S. Hasegawa, Y. Hayasaki, V. Mizeikis, R. Buividas, and S. Juodkazis, "Ultrafast laser processing of materials: from science to industry," Light Sci. Appl. 5, e16133 (2016).

[6] M. Chanal, V. Y. Fedorov, M. Chambonneau, R. Clady, S. Tzortzakis, and D. Grojo, "Crossing the threshold of ultrafast laser writing in bulk silicon," Nat. Commun. 8, 773 (2017).

[7] A. Ródenas, M. Gu, G. Corrielli, P. Paiè, S. John, A. K. Kar, and R. Osellame, "Three-dimensional femtosecond laser nanolithography of crystals," Nat. Photonics (2018).

[8] D. Puerto, W. Gawelda, J. Siegel, J. Bonse, G. Bachelier, and J. Solis, "Transient reflectivity and transmission changes during plasma formation and ablation in fused silica induced by femtosecond laser pulses," Appl. Phys. A 92, 803-808 (2008).

[9] K. J. Waedegaard, D. B. Sandkamm, A. Mouskeftaras, S. Guizard, and P. Balling, "Probing ultrashortpulse laser excitation of sapphire: From the initial carrier creation to material ablation," Europhys. Lett. 105, 47001 (2014).

[10] L. Gallais and S. Monneret, "Time-resolved quantitative-phase microscopy of laser-material interactions using a wavefront sensor," Opt. Lett. 41, 3245 (2016).

[11] K. Sokolowski-Tinten, J. Bialkowski, A. Cavalleri, D. von der Linde, A. Oparin, J. Meyer-ter-Vehn, and S. I. Anisimov, "Transient States of Matter during Short Pulse Laser Ablation," Phys. Rev. Lett. 81, 224227 (1998).

[12] W. Gawelda, D. Puerto, J. Siegel, A. Ferrer, A. Ruiz de la Cruz, H. Fernández, and J. Solis, "Ultrafast imaging of transient electronic plasmas produced in conditions of femtosecond waveguide writing in dielectrics," Appl. Phys. Lett. 93, 121109 (2008).

[13] M. Garcia-Lechuga, "Técnicas de imagen con resolución temporal aplicadas al procesado de materiales con pulsos láser ultracortos," Universidad Autonoma de Madrid (2017).

[14] J. Bonse, S. Hohm, S. V. Kirner, A. Rosenfeld, and J. Kruger, "Laser-Induced Periodic Surface StructuresA Scientific Evergreen," IEEE J. Sel. Top. Quantum Electron. 23, 9000615 (2017).

[15] M. Garcia-Lechuga, J. Siegel, J. Hernandez-Rueda, and J. Solis, "Femtosecond laser ablation of dielectric materials in the optical breakdown regime: Expansion of a transparent shell," Appl. Phys. Lett. 105, 112902 (2014).

[16] M. Garcia-Lechuga, J. Siegel, J. Hernandez-Rueda, and J. Solis, "Imaging the ultrafast Kerr effect, free carrier generation, relaxation and ablation dynamics of Lithium Niobate irradiated with femtosecond laser pulses," J. Appl. Phys. 116, 113502 (2014).

[17] M. Garcia-Lechuga, J. Solis, and J. Siegel, "Key stages of material expansion in dielectrics upon femtosecond laser ablation revealed by double-color illumination time-resolved microscopy," Appl. Phys. A 124, 221 (2018).

[18] S. S. Mao, F. Quere, S. Guizard, X. Mao, R. E. Russo, G. Petite, and P. Martin, "Dynamics of femtosecond laser interactions with dielectrics," Appl. Phys. A 79, 1695-1709 (2004).

[19] M. Garcia-Lechuga, J. Solis, and J. Siegel, "Key stages of material expansion in dielectrics upon femtosecond laser ablation revealed by double-color illumination time-resolved microscopy," Appl. Phys. A Mater. Sci. Process. 124, 1-8 (2018).

[20] S. I. Anisimov, N. A. Inogamov, A. M. Oparin, B. Rethfeld, T. Yabe, M. Ogawa, and V. E. Fortov, "Pulsed laser evaporation: equation-of-state effects," Appl. Phys. A Mater. Sci. Process. Process. 69, 617-620 (1999).

[21] M. Garcia-Lechuga, J. Solis, and J. Siegel, "Melt front propagation in dielectrics upon femtosecond laser irradiation: Formation dynamics of a heat-affected layer," Appl. Phys. Lett. 108, 171901 (2016). 
[2] J. Hernandez-Rueda, J. Siegel, M. Garcia-Lechuga, and J. Solis, "Femtosecond laser-induced refractive index changes at the surface of dielectrics: quantification based on Newton ring analysis," J. Opt. Soc. Am. B 31, 1676 (2014).

[23] D. Puerto, M. Garcia-Lechuga, J. Hernandez-Rueda, A. Garcia-Leis, S. Sanchez-Cortes, J. Solis, and J. Siegel, "Femtosecond laser-controlled self-assembly of amorphous-crystalline nanogratings in silicon," Nanotechnology 27, 265602 (2016).

[24] Y. Fuentes-Edfuf, M. Garcia-Lechuga, D. Puerto, C. Florian, A. Garcia-Leis, S. Sanchez-Cortes, J. Solis, and J. Siegel, "Coherent scatter-controlled phase-change grating structures in silicon using femtosecond laser pulses," Sci. Rep. 7, 4594 (2017).

[25] M. Garcia-Lechuga, D. Puerto, Y. Fuentes-Edfuf, J. Solis, and J. Siegel, "Ultrafast Moving-Spot Microscopy: Birth and Growth of Laser-Induced Periodic Surface Structures," ACS Photonics 3, 19611967 (2016).

[26] J. F. Young, J. S. Preston, H. M. Van Driel, and J. E. Sipe, "Laser-induced periodic surface structure. II. Experiments on Ge, Si, Al, and brass," Phys. Rev. B 27, 1155-1172 (1983).

\section{Introduction}

Femtosecond (fs) lasers have proven to be key tools for inducing modifications in transparent materials. Focused to small spots, they offer the possibility of highly controlled and localized energy deposition (submicrometer scale at surface or in the bulk) that is exploited today in various precision applications, including for instance ocular surgery [1] or innovative material micromachining [2,3].

These unique properties, together with the advent of new laser technologies and therefore new potential applications, have triggered strong interest in the study of the mechanisms leading to material modification, giving birth to a steadily growing research community over the last decades. Experimental and theoretical research [4] has allowed drawing a general picture of fs-laser dielectric excitation, which can be summarized as follows. The absorption process is triggered by the generation of free carriers induced by the intense laser electric field, initiated by multiphoton and/or tunnelling ionization from the valence to the conduction band, and subsequently enhanced by impact ionization. Later, a significant part of the energy of the free electron sub-system is transferred to the lattice, on a characteristic time scale that is longer than the pulse duration. If the absorbed energy exceeds a given threshold, the material melts and expands due to the high local temperature and pressure induced. If the laser beam is focused at the surface, material ablation can occur, a process of high interest for industrial material micro-machining [5]. When the beam is focused inside the bulk of the material, changes in the structural/optical properties can be induced and corresponding structures can be written in three dimensions [6,7].

Hence, due to the important role of the free electron density in the modification process, the characterization of its temporal evolution is a topic of interest. The use of all-optical pump-probe strategies has been proved very efficient in this field [8-10], taking advantage of the transient optical changes induced by the free carriers density evolution. Additionally, imaging techniques such as time-resolved microscopy have proved to provide complementary information, proving access to aspects not only related with the electronic excitation but with material transformation. This technique offers a relatively easy interpretation of the results and had a tremendous impact on the understanding of material transformation upon femtosecond laser irradiation, as in the case of ablation dynamics in metals and semiconductors [11] or for the formation of waveguides on transparent materials [12].

However, a full comprehension of the underlying mechanisms that lead to the final material modification is still incomplete. This summary of Garcia-Lechuga's thesis [13] briefly explains the advances made in the understanding of different phenomena as: the relative role of the excitation mechanism in dielectrics (multiphoton versus impact ionization), the ablation dynamics in dielectrics compared with metals and semiconductors, the study of sub-ablative material modification leading to the formation of a heat affected zone and the complex transformation dynamics triggered in semiconductors upon irradiation with multiple pulses (basis of the formation of laser-induced periodic surface structures - LIPSS [14]).

The article is structured as follows. In section 2, the irradiation set-up and the different variants of timeresolved microscopy used and developed in the thesis are explained. In section 3 , the main results and discussions of the thesis are summarized, grouped in three parts. Part one includes results on the excitation and ablation dynamics on lithium niobate, a crystalline dielectric material. Part two summarizes results obtained on the formation dynamics of a heat affected layer in a phosphate glass, demonstrating the 
importance of thermal effects during femtosecond laser material processing. In part three, the fabrication of amorphous-crystalline LIPSS in silicon and its formation dynamics by a novel time-resolved strategy is described. The article ends with some conclusions.

This article does not contain a dedicated review of the broad range of time-resolved imaging techniques that was included in the thesis. This review helps to better situate the motivations and challenges for the development of advanced imaging techniques aimed at improving our comprehension of laser-matter interaction mechanisms in the field of laser processing. We encourage readers interested in this topic to refer to chapter 2 of Garcia-Lechuga's thesis [13].

\section{Experimental methods}

This thesis was performed on the laboratories of the Laser Processing Group of the Instituto de Óptica "Daza de Valdés" - CSIC. The laser system used is a Ti:Sapphire femtosecond amplifier (Spitfire Pro, Spectra Physics) which produces pulses of 120 fs FWHM (Full Width at Half Maximum) centered at $800 \mathrm{~nm}$ wavelength at a repetition rate of $1 \mathrm{kHz}$. Figure 1 shows the experimental set-up. A mechanical shutter allows selecting a single pulse, which is split into two beams: pump and probe. The pump pulse is spolarized and focused on the sample surface at an angle of $52^{\circ}$ exciting an elliptical region. The pulse energy is controlled by a combination of a half wave plate and a polarizing cube beam splitter, and is monitored with a calibrated reference photodiode (PD). The pump pulse passes through a motorized optical delay line, enabling the control of the temporal delay between pump and probe pulses, with a resolution of a few tens of femtoseconds.

The probe pulse wavelength used for illumination is either $800 \mathrm{~nm}$ or $400 \mathrm{~nm}$. The probe beam is directed onto the optical axis of an imaging microscope by means of a 50-50 beam splitter with a broad spectral working range (350-1100 nm). In order to generate a large illuminated field at the sample surface, the beam is focused before the entrance of the microscope objective. The light reflected at the sample surface is collected by the objective and imaged onto a charge-coupled device (CCD) camera, employing a tube lens (TL).

Illumination (probing) at $400 \mathrm{~nm}$ is produced by frequency doubling the fundamental laser wavelength using a beta barium borate crystal (BBO), as sketched in figure 1(a). A prism compressor was used in order to pre-compensate the group velocity dispersion experienced by the probe pulse upon its pass through the optical elements of the set-up. By optimizing the distance of the two prisms in the compressor, probe pulses of approx. 150-fs duration at the sample position were achieved. This experimental variant is completed with a band-pass filter placed in front of the CCD, blocking scatter from the pump pulse $(800 \mathrm{~nm})$.

(a)

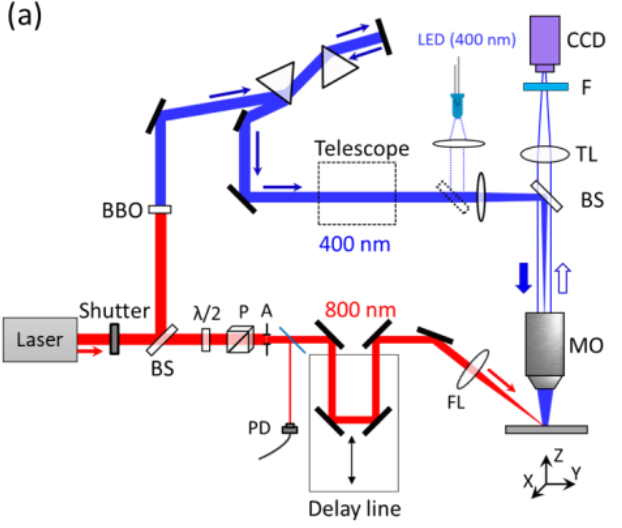

(b)

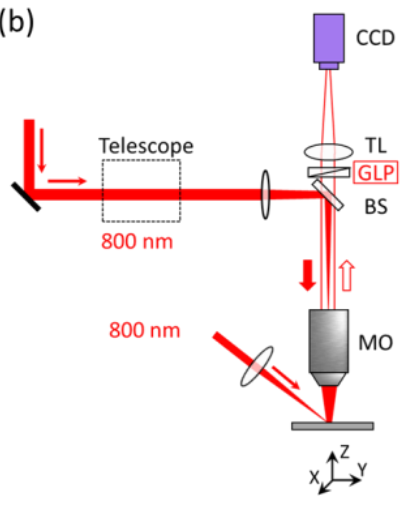

(c)

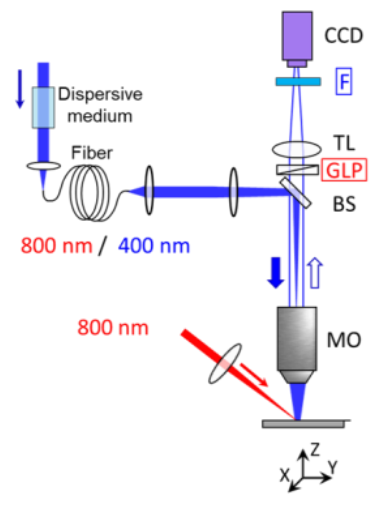

Fig. 1. Sketch of the irradiation and different configurations of the femtosecond microscopy set-up. (a) Configuration for illumination at $400 \mathrm{~nm}$. (b) Configuration for illumination at $800 \mathrm{~nm}$. (c) Configuration for long time delays (>10 ns) either with $400 \mathrm{~nm}$ or 800 nm illumination. BS: beam splitter; $\lambda / 2$ : half-wave plate; P: polarizer; A: aperture; PD: fast photodiode; FL: focusing lens; BBO: frequency doubling crystal; MO: microscope objective; TL: tube lens; F: band-pass filter; GLP: Glan-Laser calcite polarizer; CCD: charge-coupled device (camera).

Illuminating (probing) at $800 \mathrm{~nm}$ is challenging, since discrimination between pump and probe light cannot be done spectrally. In this case, scattered pump light filtering is performed by polarization discrimination. To this end, a high-extinction-ratio Glan-Laser calcite polarizer is placed just before the tube lens, as sketched in figure 1(b). Independently of the illumination wavelength, it was tested that the probe beam 
does not contribute to the final modification produced in the material, neither on its own nor in combination with the pump pulse.

To study laser-induced effects occurring on the microsecond time scale (e.g. thermal effects), an optical delay configuration in air is not practicable since it would require hundreds of meters of optical path. To this end, a plug-and-play optical fiber stage was developed, based on multimode fiber patchcords with moderate energy attenuation over a broad optical bandwidth $(250-1200 \mathrm{~nm})$, as sketched in figure 1 (c). In order to avoid white light generation inside the fiber or damage upon focusing the probe pulse into the fiber, it was necessary to perform temporal pulse stretching by inserting a $15 \mathrm{~cm}$ long borosilicate glass block. Fiber patchcords with different lengths can be connected at demand, which provides this system with flexibility to cover a delay window from 100 fs up to 0.5 microseconds.

The experimental procedure, for whatever illumination (probing) strategy, is as follows. Each time-resolved image was recorded upon irradiation with a single laser pulse, at a fixed time delay between pump and probe pulse and irradiating an unexposed material surface. Before irradiation, an image of the unexposed surface is recorded upon illumination with a probe pulse, which is used to normalize the image recorded upon pump excitation by means of image division pump/probe. That way, the normalized images provide information on the transient changes in reflectivity, with the relative reflectivity $\left(R_{r e l}\right)$ of the non-excited surface being equal to 1 . The spatial dimension of the images together with the Gaussian energy profile of the laser beam, allows accessing the material response under different local fluences after a single pulse irradiation with a peak fluence equal to $F_{0}$. For obtaining the corresponding local fluence at a given distance from the center $(\Delta \mathrm{x})$ the following formula is used $F(\Delta x)=F_{0} e^{-2 \Delta x^{2} / \omega_{x}^{2}}$.

\section{Results and discussion}

\section{3.a. Excitation and ablation dynamics of lithium niobate}

This section summarizes the main aspects of the study of the ablation dynamics of lithium niobate upon irradiation with single ultrashort pulses. More details on the results and its analysis can be found in chapter 4 of the thesis [13] and in publications [15-17].

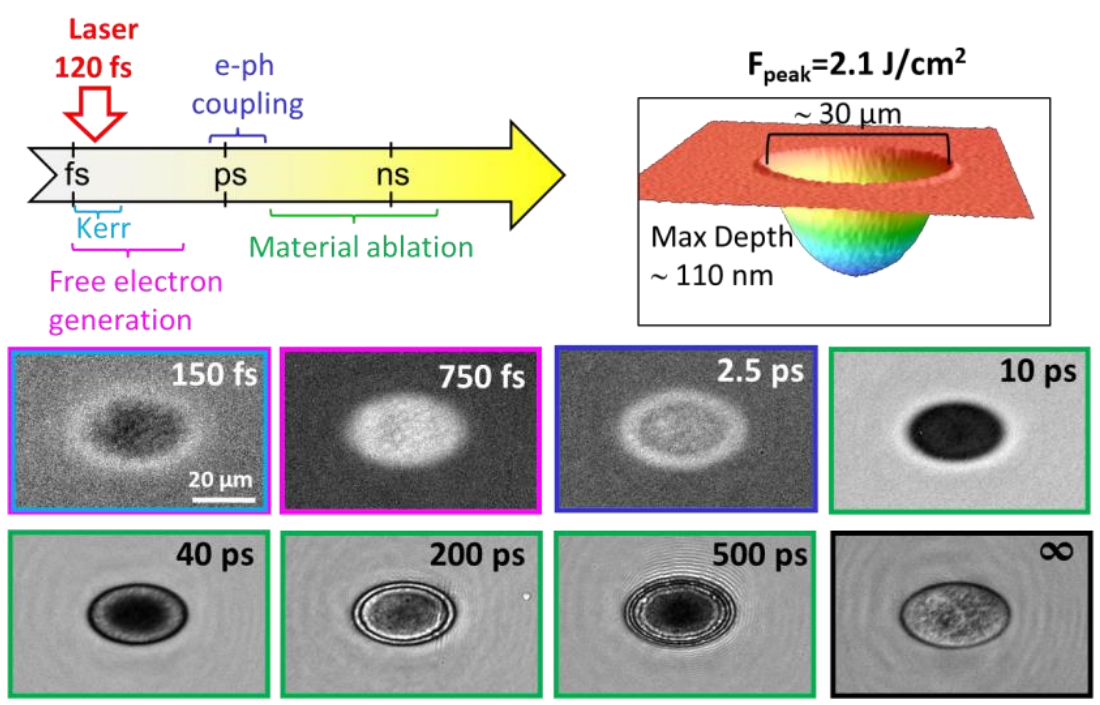

Fig. 2. (Top) Sketch of the characteristic time scales of the different mechanisms involved in the material transformation together with the permanent crater topography profile produced after a single-pulse irradiation $\left(\lambda_{\text {pump }}=800 \mathrm{~nm}\right.$, pulse duration $120 \mathrm{fs}$ and peak fluence $\left.2.1 \mathrm{~J} / \mathrm{cm}^{2}\right)$. (Bottom) Time-resolved surface reflectivity images $\left(\lambda_{\text {probe }}=400 \mathrm{~nm}\right)$ of lithium niobate at different time delays times (see labels) after exposure to a single pump pulse incident at $52^{\circ}$. The figure labeled as infinite (several seconds after irradiation) corresponds to the permanent modification. Each image is framed with a given colour, indicating the corresponding dominant physical mechanism at that time scale, as indicated on the sketch.

Figure 2 shows a sketch summarizing the different phenomena and their characteristic timescales involved in the transformation dynamics of lithium niobate under irradiation conditions above the threshold of ablation ( $40 \%$ above threshold), leading to crater formation with a maximum depth of $110 \mathrm{~nm}$. This summary is based on the observations and characterizations of time-resolved images of the surface of lithium niobate recorded at different time delays, also shown on figure 2. The first change observed on ultrashort timescales is an increase of reflectivity in a region where the local fluence is lower than the 
fluence threshold of ablation (see the ring appearing in the image at a delay on $150 \mathrm{fs}$ in figure 2). This effect is transient ( $<500 \mathrm{fs}$ ) and corresponds to the optical Kerr effect, associated to the interaction of the strong electric field with bound electrons in the valence band. The observation of this nonlinear optical effect, appearing only in the presence of a strong electric field, enables the determination of the arrival of the pump pulse at the sample surface with a resolution of tens of femtoseconds. This determination of the zero delay between the pump and probe beam is of major importance for a precise characterization of the excitation dynamics, being one of the former limitations of time-resolved microscopy compared with other techniques [18].

Another effect occurring on a similar time scale is the generation of free electrons. The reflectivity changes associated to it dominate at local fluences above the fluence threshold of modification, corresponding to the centre of the images shown in Figure 2. In that central region the reflectivity first decreases and then rapidly increases because of the generation of a high density of free electrons, reaching its maximum at a delay 600 fs. This indicates that free electron generation continues even if the excitation pulse intensity is low/absent (pulse duration 120 fs FWHM), demonstrating the contribution of impact ionization to free electron excitation. Using a model that relates the change of reflectivity and the free electron density (Drude model), the maximum electron density reached under these irradiation conditions is approx. $7 \cdot 10^{22} \mathrm{~cm}^{-3}$.

On the scale of a few picoseconds after irradiation, the reflectivity decreases as a consequence of a decrease in the free electron density and absorption effects due to the formation of a layer of material at high pressure and temperature. From tens of picoseconds onward, the formation of transient Newton rings was observed. This phenomenon is associated to the interference pattern (constructive and destructive interference) formed by the probe beam reflection at the front and rear surface of an expanding layer of ablated material. These Newton rings were previously observed in metals and semiconductors, but never reported for a dielectric material. The universality of this phenomenon has been further demonstrated by reporting the formation of rings in two other dielectric materials (sapphire and SF57 glass) when irradiated with fluences above the ablation threshold [15].

(a)

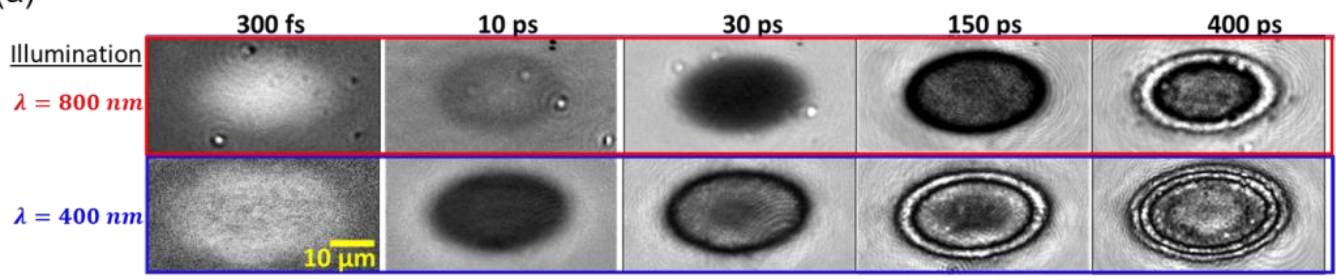

(b)
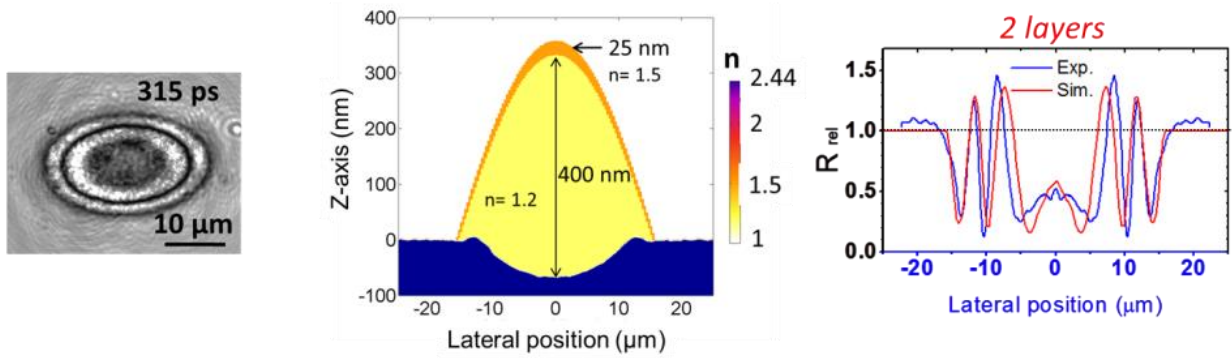

Fig. 3. (a) Representative time-resolved reflectivity images of a lithium niobate surface upon single-pulse irradiation $\left(\lambda_{\text {pump }}=\right.$ $800 \mathrm{~nm}, 120$-fs pulse duration) with a peak fluence of $2.0 \mathrm{~J} / \mathrm{cm}^{2}$. Two different illumination wavelengths (400 nm and $\left.800 \mathrm{~nm}\right)$ were used for recording. (b) (left) Image recorded at a delay of $315 \mathrm{ps}$ after excitation (peak fluence of $2.0 \mathrm{~J} / \mathrm{cm}^{2}$ ). (center) Cross section of a simulated optical layer system of ablating material corresponding to this time delay. (left) Experimental and simulated reflectivity profiles for a delay of 315 ps. The editing of the figure is original, however, some of the images on (a) have been already published in [19]

The interference nature of these rings is confirmed by recording images with an illumination wavelength of $800 \mathrm{~nm}$, as shown in Figure 3(a). As an example, a total of 8 maxima and minima rings are observed for a delay of 400-ps when illuminating with $400 \mathrm{~nm}$. In contrast, a total of 4 rings are observed when illuminating with $800 \mathrm{~nm}$ for the same delay. This implies that the number of rings is directly correlated 
with the optical thickness of the expanding layer. The characterization of the number of rings allowed to determine the expansion velocities. For fluences $30 \%$ above the modification threshold, velocities of $\sim 1000$ $\mathrm{m} / \mathrm{s}$ were obtained.

Additionally, the evolution of the reflectivity of Newton rings has been simulated by using a multilayer optical model. It has been observed that up to the 100-ps scale after irradiation the reflectivity simulation is consistent with the expansion of a single homogeneous layer. However, for longer delays, a two-layer system needs to be considered, containing a very thin top layer (with a higher refractive index), as represented on figure 3 (b). Such shell-like configuration had previously been proposed on the basis of calculations with a hydrodynamic model in metals [20], but never been demonstrated experimentally.

\section{3.b. Heat-affected layer formation on a glass}

This section summarizes the main aspects of the formation dynamics of a heat affected layer in glasses when irradiating them with single ultrashort pulses. More details on the results and analysis can be found in chapter 5 of the thesis [13] and in publication [21].

Figure 4 (a) shows a topographic elevation of about $40 \mathrm{~nm}$ at the surface of a phosphate glass after irradiating with a fluence slightly below the ablation threshold. This permanent elevation is caused by the decrease of material density in the irradiated region, which also induces a change of other properties in a thin layer underneath. By analysing the ring interference pattern observed by optical microscopy also shown in figure 4 (a), a maximum layer thickness of $720 \mathrm{~nm}$ can be determined. The observation of this permanent ring pattern was also observed in a number of materials [22], indicating the importance of thermal phenomena after femtosecond laser irradiation.

The observation of this effect with thermal origin in the subablative regime allowed to study the formation dynamics of the heat-affected layer by time-resolved microscopy, without the influence of ablative effects that would screen its observation. The study is performed for a very wide time window, from few hundreds of femtoseconds $\left(10^{-13} \mathrm{~s}\right)$ to hundreds of nanosecond $\left(10^{-7} \mathrm{~s}\right)$, by using the fiber-optic delay line described in section 2. Figure 4 (b) shows a sequence of images covering this time window. For a delay of $750 \mathrm{fs}$, an increase in reflectivity caused by the generation of a high density of free electrons $\left(\sim 1 \cdot 10^{22} \mathrm{~cm}^{-3}\right)$ can be observed. The subsequent decrease in reflectivity (images at 8 ps and 20 ps time delay) and its subsequent relatively slow evolution indicates that the mechanism of material modification differs from the ablation process.

(a)

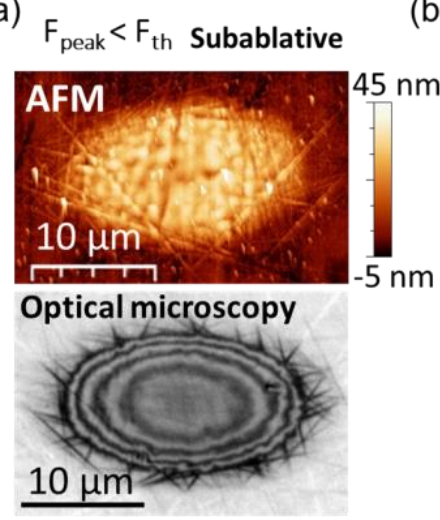

(b)
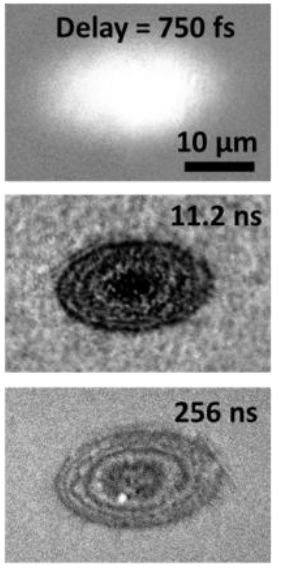
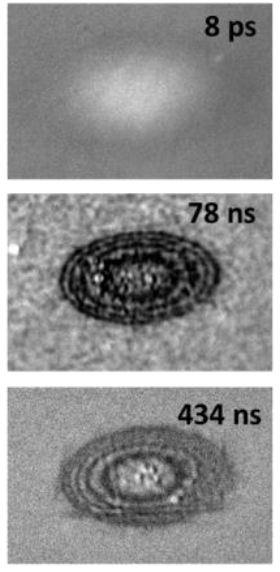

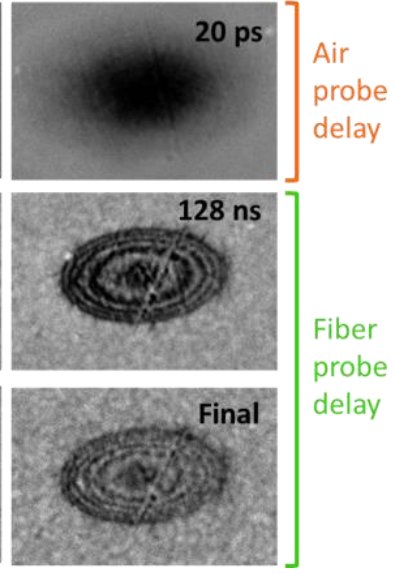

Fig. 4. (a) Image obtained with atomic force microscopy (AFM) and ex-situ optical microscopy image with illumination at 460 nm after irradiation with a single $120 \mathrm{fs}$ pulse with a peak fluence of $3.7 \mathrm{~J} / \mathrm{cm}^{2}$. (b) Time-resolved images captured upon irradiation (delay indicated in each image). All the images recorded by illuminating through an optical fiber are represented with the same image contrast. The editing of the figure is original, however, some of the images on (a) and (b) have been already published in [21].

By exploring the evolution of the reflectivity of the material surface at time delays between $10 \mathrm{~ns}$ and 0.5 $\mu \mathrm{s}$ by propagating the probe beam through fibers of different lengths, the formation and evolution of a dynamic ring pattern has been observed. These transient Newton rings have been associated with the presence of a thin layer of molten material, which increases in thickness as the melt front propagates in depth. 
From the analysis of the images and optical modelling, the temporal evolution of the maximum thickness and refractive index of the melt layer is characterized. This data, represented in figure 5 (a), demonstrates that the propagation of this front follows an exponential function that reaches a maximum depth of $720 \mathrm{~nm}$, approximately $1 \mu \mathrm{s}$ after excitation. The propagation velocity, extracted from a derivation and represented in figure 5 (b), follows a decreasing exponential trend, taking values of $1 \mathrm{~m} / \mathrm{s}, 0.3 \mathrm{~m} / \mathrm{s}$ and $0.1 \mathrm{~m} /$ at $5 \mathrm{~ns}$, $300 \mathrm{~ns}$ and $600 \mathrm{~ns}$, respectively. These values are much lower than the typical values of sound propagation in glass, confirming the propagation of a thermal front rather than a shock wave.

The temporal evolution of the refractive index of the molten material layer is also characterized. It is observed that the refractive index change is always negative. This can be understood by taking into account that the material undergoes a process of density decrease (rarefaction), a consequence of the heating and melting process of the material, consistent with the surface swelling observed in figure 4(a). The permanent change in the refractive index is characterized to be equal to a $-1.4 \%$, which is consistent with the permanent decrease of the density in the irradiated region, causing the topographic elevation observed experimentally.

(a)

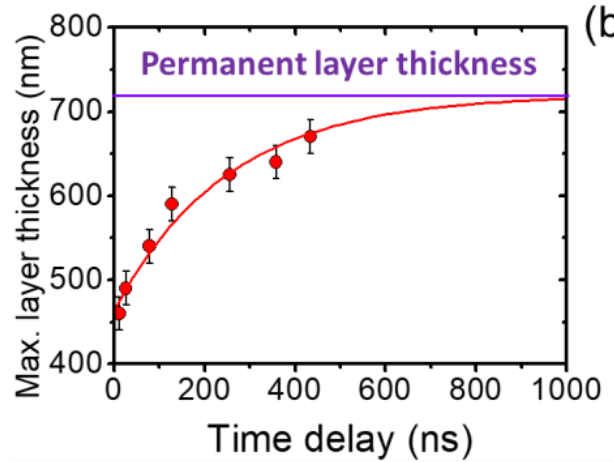

(b)

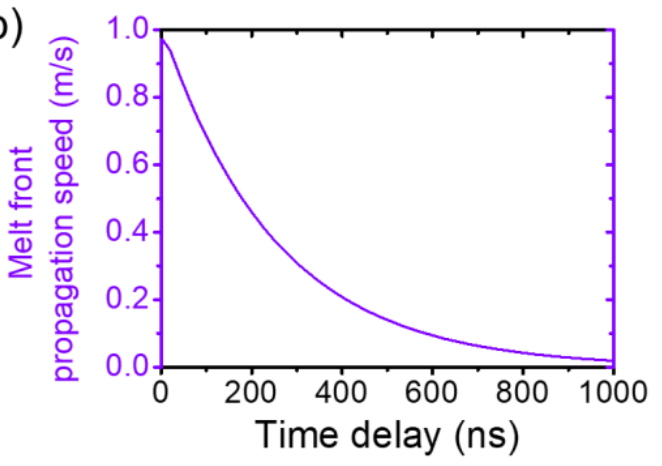

Fig. 5. (a) Evolution of the thickness of the molten layer as a function of the time delay after single-pulse irradiation of a phosphate glass. The red solid curve is a fit of the experimental points to an exponential function. (b) Propagation speed of the melting front as a function of time delay after irradiation.

These results corroborate the importance of thermal effects in laser processing of materials with ultrashort pulses. The study of thermal processes after excitation provides especially relevant information for the processing of materials with multiple pulses, since depending on the temporal separation between pulses (inverse to the repetition frequency), consecutive pulses can impinge on an already resolidified material or a still viscous material, which can induce different final modifications.

\section{3.c. Fabrication and formation dynamics of laser-induced periodic surface structures in silicon}

In this section the main aspects of the fabrication and time-resolved formation of amorphous-crystalline laser-induced periodic surface structures in silicon (a-c LIPSS) are summarized, which are formed after irradiation with multiple ultrashort pulses. More details on the results and analysis can be found in chapter 6 of the thesis [13]. Additionally, details on the LIPSS fabrication process can be found in publications $[23,24]$ and details on the time-resolved measurements in publication [25].

First, the fabrication feasibility of periodic structures in silicon $\langle 100\rangle$ without inducing ablation in the material is demonstrated. These structures consist of alternating stripes of amorphous and crystalline material. This local phase transformation of the material was characterized by optical microscopy (the amorphous phase shows a higher reflectivity than the crystalline phase) and by Raman spectroscopy with micrometer resolution.

In a first step, these periodic structures were generated by static irradiations, finding an optimum dose (number of pulses and fluence) for their formation. Secondly, dynamic irradiation, by scanning the beam over the sample, allowed to generate periodic structures along the scan line. It should be mentioned that the scanning should follow the same direction as the polarization of the incident beam in order to generate high quality stripes. This requirement is linked to the local field enhancement caused by the interaction of 
the incident field with a surface wave propagating in a direction perpendicular to the fringes generated by previous pulses.

(a)

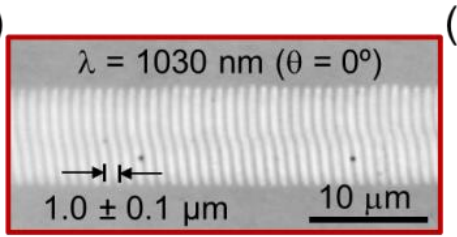

(b)

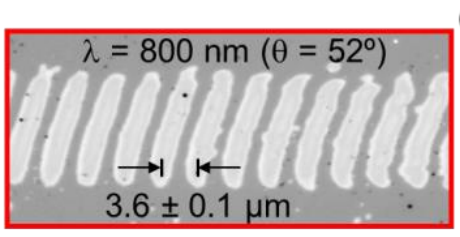

(c)

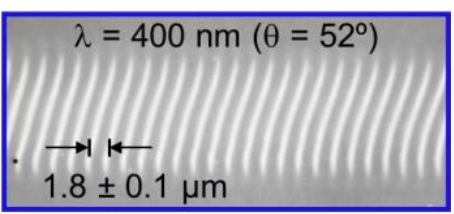

Fig. 6. Optical microscopy images ( $460 \mathrm{~nm}$ illumination) of a-c LIPSS in silicon generated with different lasers (370 fs, $500 \mathrm{kHz}$ and $1030 \mathrm{~nm}$ for (a) and $120 \mathrm{fs}, 100 \mathrm{~Hz}, 800 \mathrm{~nm}$ (b) or $400 \mathrm{~nm}$ (c)) and different irradiation conditions (normal incidence for (a) and an angle of $52^{\circ}$ for (b) and (c)). In all three cases the beam was horizontally polarized (p-polarized). The editing of the figure is original, however, the microscope images have been already published in [23].

The fabrication of $a-c$ LIPSS was performed by irradiating with three different wavelengths $(1030 \mathrm{~nm}, 800$ $\mathrm{nm}$ and $400 \mathrm{~nm})$ and two different angles of incidence $\left(0^{\circ}\right.$ and $\left.52^{\circ}\right)$. The results are displayed in figure 6 , showing that the periodicity depends on the angle of incidence and the excitation wavelength, fitting well with theoretical calculations applying a basic scattering model [26].

Secondly, a strategy based on time-resolved microscopy as sketched in figure 7 (a), allowed simultaneously to fabricate $a-c$ LIPSS and assess their spatial and temporal formation dynamics. The method is based on a discrete sample displacement strategy (moving-spot) before each individual irradiation pulse. The irradiation fluence and displacement were adjusted to ensure both high quality structure formation and constant excitation and sampling conditions. These constant conditions allow to have access to the evolution of the reflectivity of the structures finally generated and to associate it to the different processes of excitation and modification of the material.

In this particular case, the strategy was applied to the study of the a-c LIPSS in silicon generated upon irradiation with pulses a wavelength of $800 \mathrm{~nm}$, a duration of $120 \mathrm{fs}$ and an incident angle of $52^{\circ}$. Those $a-c$ LIPSS were characterized to have a periodicity equal to $3.5 \mu \mathrm{m}$ and an amorphous layer thickness of about $50 \mathrm{~nm}$. Figure 7 (b) shows a few time-resolved images recorded at different timescales following the moving-spot strategy.

(a)

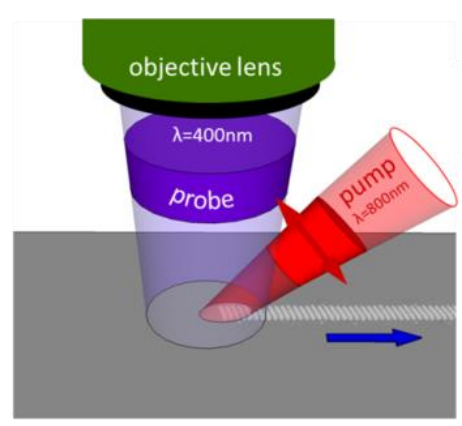

(b)

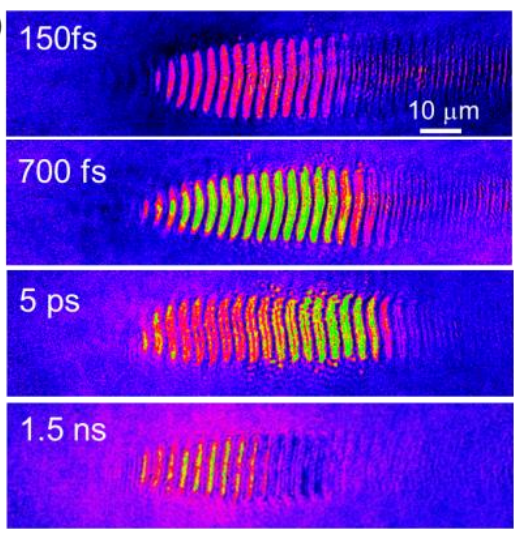

(c)

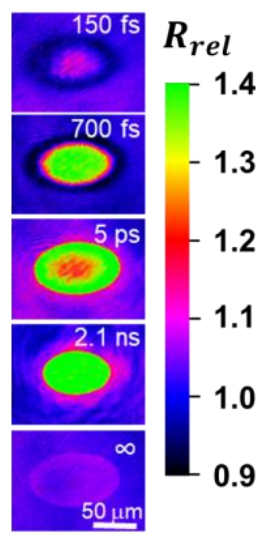

Fig.7. (a) Schematic of the ultrafast-moving spot microscopy technique. A p-polarized excitation pulse irradiates the surface of the material on which several previous pulses have generated LIPSS. An illumination (probe) pulse passing through the microscope objective reaches the surface with a certain delay. (b) False-color time-resolved reflectivity images of the silicon surface during laser writing of amorphous fringes. The irradiation beam is a pulse at $800 \mathrm{~nm}, 120$-fs pulse duration and a peak fluence of $140 \mathrm{~mJ} / \mathrm{cm}^{2}$.

The images show the relative reflectivity changes with respect to the fringe structure before irradiation (used for normalization). (c) Selected time-resolved images of single-pulse excitation of nonstructured c-Si for comparison (peak fluence $240 \mathrm{~mJ} / \mathrm{cm}^{2}$ ). The editing of the figure is original, however, some of the images have been already published in [25]

In order to associate the observations of figure 7(b) to different physical processes, a reference experiment is performed by irradiating silicon with a single pulse below the ablation threshold, as shown in figure 7(c). The initial ultrafast response is the formation of a high density of free electrons, which can be appreciated by an increase of the reflectivity at ultrashort time delays. As a result of the high level of electron excitation, the material undergoes a non-thermal melting process. This results in a high material reflectivity associated 
with the formation of a molten silicon layer, with a higher reflectivity than solid silicon. Few picoseconds after the irradiation a decrease in the reflectivity occurs, which can be attributed to an overheating of the molten silicon layer, well above the melt temperature. Finally, on a scale of a few nanoseconds, rapid resolidification of the material occurs, leading to partial amorphization of the material, as observed in the image labelled as $\infty$ in figure 7 (c).

The results observed during the formation dynamics of the $a-c$ LIPSS are consistent with the processes observed in this single-shot reference experiment. In particular, the results unambiguously show that no melting occurs between amorphous fringes, which corroborates the LIPSS formation mechanism is based on a local electric field enhancement due to the interference of incident light and a surface wave. Finally, it should be noted that the moving-spot strategy can be applied to the dynamical study of other types of LIPSS, which may bring more clarity to the still open debate on the formation mechanisms of such structures.

\section{Conclusions}

In this $\mathrm{PhD}$ thesis different strategies based on time-resolved microscopy have been developed and applied to the study of transient and permanent changes at the surface of materials occurring upon femtosecond laser excitation. By recording and analysing images at different time scales in combination with theoretical modelling, different mechanisms leading to material modification have been characterized in detail.

These techniques have been applied to various case studies in the frame of laser processing, involving ablation regimes (study of the ablation dynamics of lithium niobate) or sub-ablation regimes, either on a single pulse basis (formation of a heat affected layer in phosphate glass) or multi-pulse basis (formation of amorphous-crystalline LIPSS in silicon).

As a general conclusion, the results presented in this thesis demonstrate the huge potential of time-resolved pump-probe microscopy for revealing the dynamics of a multitude of material modification processes upon femtosecond laser excitation. Apart from providing more insight in the physical mechanisms related to laser-matter interaction, they constitute a powerful tool for identifying optimum conditions for technological applications of femtosecond laser structuring.

\section{Acknowledgements}

This work has been supported by the LiNaBioFluid project (H2020-FETOPEN-2014-2015RIA, grant 665337) of the European Commission as well as by the Spanish Ministry of Economy and Competiveness through research projects (TEC2011-22422 and TEC2014-52642-C2-1-R) and an FPU fellowship (Grant No. AP2012- 0217) from the Spanish Ministry of Education. Grant No. AP2012- 0217. The author warmly thanks Francisco Javier Solis Céspedes and Jan Siegel for being the supervisors of this thesis. The author thanks the support and kindness from colleagues of Laser Processing Group and Instituto de Óptica - CSIC (technical staff, administrative staff and researchers). 